\title{
La eficiencia energética en la vivienda como responsabilidad y beneficio conjunto
}

\section{Energy efficiency in housing as a shared responsibility and benefit}

\author{
Lucas Rodríguez, José Luis Fernández \\ Carrera de Arquitectura, Departamento de Geografía y Turismo, Universidad Nacional del Sur \\ arqlucasgrodriguez@gmail.com, jlfernandez.arq@gmail.com
}

\section{RESUMEN}

Las bajas calidades constructivas en la vivienda producen una demanda permanente de energía auxiliar para sostener niveles mínimos de habitabilidad, generando una elevada dependencia urbana del insumo energético, impactando significativamente sobre la matriz energética nacional. En consecuencia, se plantea a la eficiencia energética edilicia (EEE) como estrategia prioritaria de mitigación; generando beneficios para todos los actores intervinientes. Impulsados por tales inferencias, se ha trabajado en etapas concatenadas: una primera de investigación y desarrollo (a través de investigaciones en la FAU-UNLP y CONICET), una segunda etapa de difusión y capacitación (desde el CAPBA), y una propuesta de tercera etapa en formación académica (desde la UNS). A modo de conclusión se remarca que la aplicación de la EEE sobre el parque residencial requiere de la participación sinérgica del sector público y el privado, a partir de lo cual se obtienen beneficios para todos los actores intervinientes (organismos de gobierno, cuerpos colegiados, profesionales y usuarios).

\section{ABSTRACT}

The low constructive quality in home building produce a permanent demand of auxiliary energy to sustain minimum habitability standards, generating a high urban demand of energetic supply, which results in a significant impact on the national energetic matrix. Therefore, the building energetic efficiency (EEE) is proposed as a prior mitigation strategy, as it may generate benefits for all actors involved. Moved by such inferences, a work has been produced in concatenated phases: research and development being the first (through research in the FAU-UNLP y CONICET), a second phase based on promotion, dissemination and training (through the CAPBA), and a third base centered in the academic education (within the UNS). As a conclusion, the application of EEE on the residential sector requires the synergetic participation of both public and private sectors, from which benefits for all actors (government organisms, collegiate bodies, professionals and users) are obtained.

PALABRAS CLAVE: vivienda, sector residencial, eficiencia energética

KEYWORDS: Dwelling, housing, energy efficiency

FECHA DE RECEPCIÓN: 12/9/2021 | FECHA DE ACEPTACIÓN: 19/10/2021

DOI: http://dx.doi.org/10.30972/arq.0185674 


\section{INTRODUCCIÓN}

El contexto energético actual de la Argentina, así como el consumo del parque edilicio urbano existente, forman parte de una problemática vigente que necesariamente debe abordarse en forma crítica y propositiva a efectos de minimizar la demanda de recursos fundamentalmente fósiles. Si tenemos en cuenta que en Argentina la gran mayoría de la población reside en áreas urbanas, con cifras en incremento, se reconoce una demanda en alza con su consecuente incidencia en la matriz energética nacional (MEN). Esta situación se verifica actualmente en la participación de la vivienda construida (sector residencial) con relación a los otros sectores involucrados (Comercial y Público, Industrial, Transporte, Agropecuario y No Energético), con un consumo superior a la cuarta parte del total del país (BEN, 2020).

Esta demanda, ante una oferta limitada de recursos fundamentalmente fósiles, genera competencias poco benéficas a nivel país dado que en situaciones climáticas extremas se definieron prioridades de uso de ciertos insumos energéticos, como el gas natural (GN) en invierno y energía eléctrica en verano, necesitando priorizar su oferta al sector residencial para cubrir las necesidades básicas de habitabilidad. Por lo que consecuentemente, se comprometió al sector industrial a la sustitución de fuentes menos competitivas y más contaminantes. Si se considera una proyección de crecimiento sostenida en el tiempo del sector residencial urbano, dado que en las últimas décadas la producción edilicia formal se ha incrementado fuertemente duplicando en el año 2009 el promedio anual de la superficie construida respecto de 1991 (INDEC, 2010)-, dicha situación ampliaría la brecha y profundizaría la competencia, maximizando paralelamente los problemas ambientales.

Teniendo en cuenta los consumos presentes en el sector residencial y entendiendo a las ciudades como estructuras energo-intensivas (DISCOLI, 2009) que dependen necesariamente de estos recursos para mantener las condiciones requeridas de confort y habitabilidad, se presenta la necesidad de generar propuestas para minimizar la demanda de climatización en forma masiva, buscando una relación más eficiente entre el consumo energético creciente y la eficiencia en el uso. Para ello se rescatan acciones para trabajar sobre el mejoramiento de las variables constructivas de la envolvente edilicia (muros, cubiertas, abertura) en el parque residencial urbano, permitiendo así establecer una relación más equilibrada entre la energía utilizada, la posible sustitución, la habitabilidad y sus consecuencias ambientales.

En esta tónica, el artículo propone describir acciones sobre conservación y eficiencia energética en el sector residencial, desarrolladas por los autores en el marco de la investigación científica y el campo profesional. Al respecto, se describe el desarrollo de dos etapas manifestadas, previéndose una tercera: i) investigación y desarrollo; ii) difusión y capacitación; y iii) aportes desde la formación de grado académico.

\section{METODOLOGÍA}

Recordamos que este trabajo se presenta desde la voluntad de compartir acciones realizadas en el marco de la eficiencia energética residencial, para reforzar el llamado a la responsabilidad de cada uno de los actores intervinientes. En consecuencia, el artículo en sí presenta una metodología descriptiva interpretativa, contrastando la realidad observada con los supuestos teóricos. A su vez, tales descripciones fueron desarrolladas a partir de las estrategias de abordaje establecidas para la tesis doctoral del Dr. Arq. Lucas Rodríguez, extendidas al proyecto de I+D CONICET (2012-2014) titulado "Desarrollo de tecnologías y pautas para el reciclado masivo de la envolvente edilicia residencial orientado al uso racional y eficiente de la energía en áreas urbanas" -PIP CONICET 112-01101-00097-, dirigido por el Dr. Carlos Discoli. A continuación, detallamos la mencionada metodología. 
La complejidad del objeto de análisis, la edilicia residencial de la ciudad de La Plata, requirió del trabajo interrelacionado de 3 niveles de abordaje (fig. 1) en 3 fases operativas (identificación y clasificación, análisis y propuesta de intervención, evaluación y resultados). Se presentó su metodología de clasificación (I), la metodología de evaluación energética (ii) y económica (iii); y la instrumentación generada (iv).

i) La identificación, selección y clasificación del parque residencial existente se trabajó a partir de distintos "niveles de abordaje", partiendo desde el estudio de la ciudad y sus áreas urbanas homogéneas, identificando las unidades edilicias que conforman tales tejidos urbanos, hasta desglosar cada una de las unidades edilicias en función de sus sistemas y componentes constitutivos. Para ello, se constituyó un sistema de Matriz de Datos (Samaja, 1994) clasificando según:

a) un nivel contextual (o nivel supra-unitario) correspondiente a la escala urbana, trabajando con mosaicos urbanos (Viegas, 2010) como herramienta de clasificación de áreas urbanas homogéneas representativas.

b) un nivel de anclaje (o nivel unitario) correspondiente a las unidades edilicias, donde se trabaja con tipologías edilicias (Rosenfeld y Czajkowski, 1992), cuya ventaja fundamental radica en su capacidad de síntesis descriptiva desde lo formal y lo tecnológico (cuantitativo) y su reconocimiento simbólico-contextual (cualitativo).

c) un nivel de componente (o nivel sub-unitario), donde se trabaja según los sistemas tecnológico-constructivos (como marco de producción material de la edilicia construida) y los elementos de las envolventes (muros, cubiertas y aberturas) y sus encuentros; los que constituyen cada uno de los componentes que al integrarse conforman las distintas tipologías residenciales (INDEC, 2010; Rodriguez, 2015).

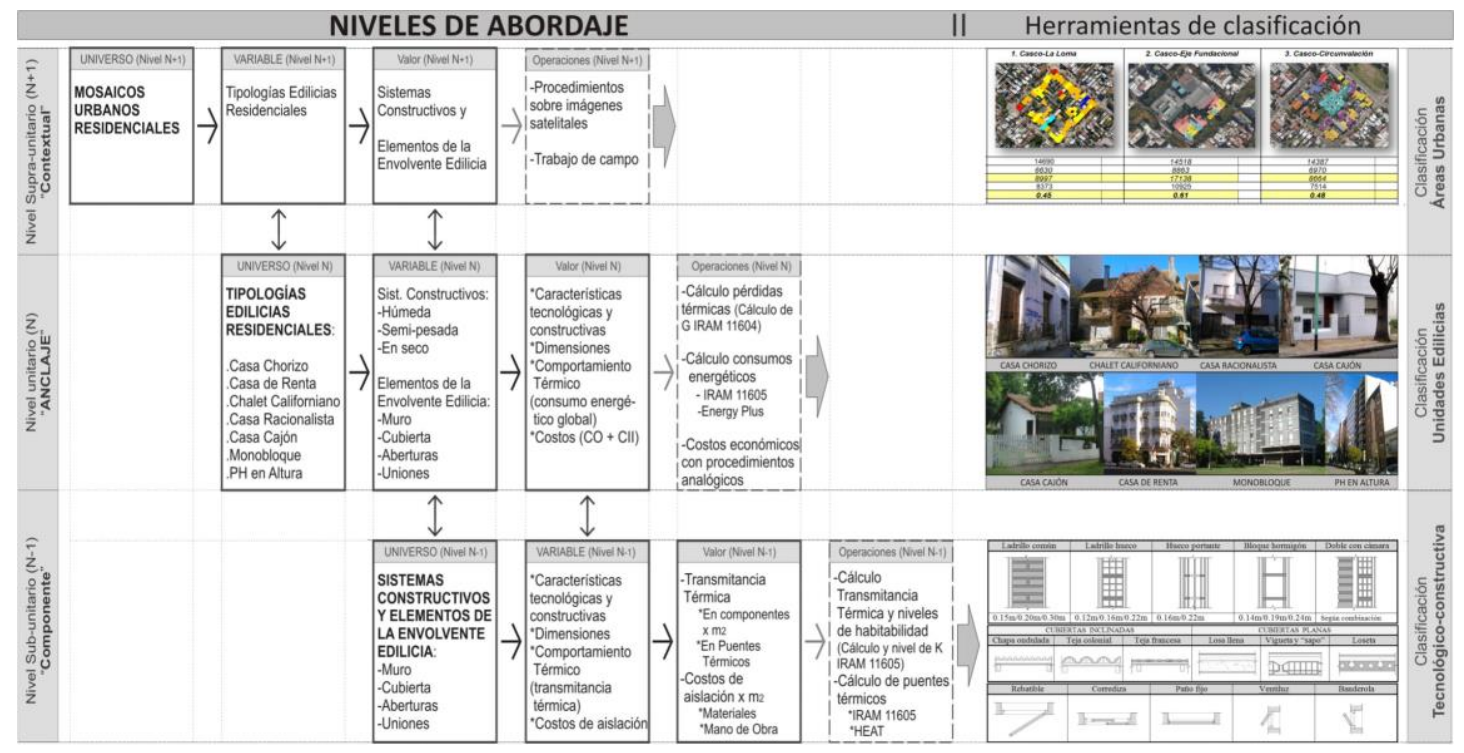

Figura 1: Sistema de matriz de datos y herramientas de clasificación para los 3 niveles de abordaje. Fuente: elaboración propia

ii) En función de la problemática planteada, los objetivos específicos y el planteo de aplicación masiva, la metodología de evaluación termo-energética debe ser ágil y fácilmente replicable. Por lo tanto, se decidió optar por los métodos de cálculo estacionario según IRAM en su serie de "Acondicionamiento térmico de edificios", cubriendo paralelamente las exigencias de la vigente Ley №13059 de la Prov. de Bs. As.

Por lo tanto, se partió de trabajar cada unidad edilicia en sus dos niveles principales: Componente y Anclaje. En el nivel de componente se analiza la transmitancia térmica (“K”) de cada uno de los 
elementos que constituyen la envolvente edilicia (muros, techos y aberturas), para el estado base y para el mejorado (según IRAM № 11601, 2001; № 11507-4, 2010). En el nivel de anclaje se analiza su comportamiento energético global (“G", según IRAM N 11604, 2001), para así obtener las demandas de energía de cada vivienda ("Q"), según IRAM No 11604, 2001) necesaria para alcanzar los niveles de habitabilidad establecidos por normativa (IRAM № 11605, 1996). Una vez estimados los valores de ahorro energético para cada tipología, se recurre al nivel contextual para generar estimaciones de disminución en la demanda, según aplicaciones masivas a las diferentes tipologías representativas de los mosaicos urbanos.

Paralelamente, a partir de reconocer las limitaciones del método de cálculo IRAM, se realizaron estimaciones de Ganancia Solar Directa (GAD) en régimen estacionario según la herramienta denominada "Modelo Energético" (GUERRERO, 1983) y simulaciones dinámicas según el software "Energy Plus" (Departamento de Energía de los Estados Unidos -DOE-) como recurso de ajuste del balance térmico. Como también se empleó el software "HEAT" (Universidad de Lund, Suecia) para el estudio particularizado de los componentes tecnológico-constructivos en sus puentes térmicos

iii) Por parte de la evaluación económica: La complejidad de los costos de materialización de las medidas de reciclado, así como la complejidad de los insumos energéticos en juego, requirieron el desarrollo de estrategias de evaluación económica y tecnológica flexibles y una instrumentación que pondere en términos relativos y específicos evaluaciones termo-económicas de las diferentes medidas a adoptar en un contexto de costos y subsidios complejo. Para ello se considera la incidencia de los costos en las distintas etapas de intervención: el costo inicial de inversión (CII) para realizar las mejoras tecnológico-constructivas, el costo operativo (CO) para afrontar el combustible consumido según equipo de climatización, el análisis de recuperación de la inversión inicial (estableciendo algunos supuestos para su estimación) y algunos escenarios de rentabilidad, como evaluación de conveniencia netamente económica.

En dicha tónica, se comprende que cada sistema propuesto debe ser analizado, indefectiblemente, desde su integración energético-económica, o sea, en su relación entre el ahorro energético logrado (con sus consecuentes ahorros en el $\mathrm{CO}$ ) y la inversión realizada (CII). Esta lógica es comprendida como una herramienta esencial y complementaria que se introduce en el sistema como rutina de evaluación rápida, permitiendo comparar y seleccionar estrategias de reciclado eficientes ante una amplia variedad de alternativas tecnológico-constructivas para la envolvente edilicia. Para ello se desarrolló una herramienta específica denominada "Índice de Eficiencia Energético-Económica" (RODRIGUEZ, 2015), la cual resulta particularmente útil para una clasificación según grado de eficiencia respecto del costo-beneficio $(\Delta E / \Delta \$)$, tanto a nivel de elementos de la envolvente, de unidades edilicias y de áreas urbanas.

iv) En relación a la instrumentación general para la integración del proceso de selección, evaluación y aplicación, se desarrolló un sistema compacto integrado que agrupa las tipologías, las tecnologías y las propuestas de reciclado general y puntual con valoraciones específicas. Dicho sistema se construyó como una plataforma interactiva en soporte digital tipo Excel que permite seleccionar los casos por medio de bibliotecas y catálogos de soluciones viables con la inclusión de análisis y valoraciones termo-físicas y técnico-económicas.

\section{DESARROLLO}

Las actividades que aquí se enuncian han dado inicio en 2009, según diversas acciones. Por motivos de extensión del presente artículo, describimos sucintamente los aportes fundamentales de las distintas etapas (investigación y desarrollo, difusión y clasificación, formación académica) para reforzar que el cumplimiento de la eficiencia energética en la vivienda es una responsabilidad y beneficio conjunto de todos los actores intervinientes.

Etapa de investigación y desarrollo 
Estas actividades fueron desarrolladas desde el Instituto de Investigaciones y Políticas del Ambiente Construido (IIPAC), FAU-UNLP en el marco de la tesis doctoral del autor y un proyecto PIP CONICET, ambos financiados por el CONICET. Paralelamente, se trabajó en sinergia con otros proyectos, acreditados por CONICET, ANPCyT y UNLP.

La investigación, en sí, se centró en la edilicia construida por ser la responsable del consumo energético actual en las incidencias mencionadas. A partir de lo cual, se debieron analizar no sólo las características técnicas válidas para lograr eficiencia termo-energética, sino también los recursos necesarios para mejorar la calidad de lo construido (reciclado edilicio o retrofitting). En consecuencia, la investigación desarrolló una metodología de sistematización de lineamientos e instrumentaciones para el reciclado en aplicación masiva, orientada a la optimización termoenergética de la envolvente en el parque residencial existente.

A su vez, se establecieron como base los mismos requisitos tecnológico-constructivos que exige la Ley 13059, según Norma IRAM, para así también aportar en una sinergia que avance hacia la mejora de las calidades higrotérmicas en las construcciones arquitectónicas; mejorando la habitabilidad en cada vivienda y reduciendo la demanda energética a nivel urbano, en el presente $\mathrm{y}$ hacia el futuro.

\section{Conclusiones de la etapa de investigación}

En cuanto a la metodología y propuestas desarrolladas en la investigación, se afirma que han podido abordar las escalas del tema; han generado una sistematización e instrumentación versátil y ágil, pudiendo operar en tiempos reducidos; y han podido evaluar los aspectos energéticos, tecnológicos y económicos de manera masiva en la escala urbana a partir de los instrumentos de clasificación y comparación propuestos. Todo lo cual nos permite generar evaluaciones generales y particulares para la proyección de escenarios de mitigación y propuestas de gestión pública, de análisis en el sector privado, revisiones en el campo profesional de la construcción y los usos vigentes de la energía en cuanto a las condiciones de habitabilidad.

En su mirada contextual, como escenario de aplicación, es fundamental reconocer que la situación energética nacional y las crecientes necesidades de importación de combustibles y energía (que significaron una fuga de divisas del $13.50 \%$ del total de las importaciones nacionales observadas en el Intercambio Comercial Argentino de 2012 (INDEC, 2013)), pueden ser tratadas y minimizadas por acciones específicas de reciclado edilicio. Las mejoras propuestas permitirían reducir significativamente la demanda en el sector residencial (del orden del 35 al $50 \%$ ), el cual insume más de un cuarto del consumo energético nacional (BEN, 2020) con cifras que no presentan una proyección de disminución. Para tal fin se requiere de decisiones estratégicas y programas políticos para mejorar la eficiencia energética en la edilicia construida, con orientación y aplicación masiva. Es claro que el actual marco energético-económico y la baja calidad térmica en cuanto a los aspectos tecnológico-constructivos demandan necesariamente respuestas concretas y sistemáticas de mejoramiento como las desarrolladas en el trabajo. Reducir la demanda disminuiría la participación del combustible importado con sus elevados costos; dado que las tarifas actuales del GN junto a la reducción en los subsidios han elevado significativamente el costo para cada usuario, cuyos precios aún se prevén en importante alza acorde al costo internacional de la energía. Y debemos recordar que la optimización desde lo tecnológico-constructivo también comprende una mejora inmediata sobre el confort interior, pudiendo alcanzar los niveles de habitabilidad dignos establecidos en la normativa nacional. Todo lo cual infiere un costo inicial que es recuperado, en condiciones nominales, en períodos del orden de los 10 años (RODRIGUEZ et al., 2017).

En síntesis, se reconoce que la eficiencia energética edilicia presenta beneficios para todos los actores involucrados, mejorando las condiciones de confort higro-térmico para el usuario; reduciendo significativamente el costo operativo y la demanda energética, con su consecuente disminución en las emisiones de gases de efecto invernadero; la posibilidad de reducir la potencia 
instalada de los equipos de climatización; y la potencialidad de aplicar tecnologías de conservación energética de fácil ejecución, con costos de inversión inicial relativamente bajos y amortizables en períodos mediatos, brindando proyecciones altamente favorables para la esfera de la gestión pública, asegurando la calidad de habitabilidad, la salud edilicia y la reducción del costo de energía auxiliar y sus subsidios.

\section{Etapa de difusión y clasificación}

Las actividades de esta etapa se desarrollan principalmente entre 2015 y 2018, en el marco de la participación de los autores desde el auspicio y promoción del Colegio de Arquitectos de la Provincia de Buenos Aires (CAPBA). Primero desde la Comisión de Desarrollo Sustentable del CAPBA1 y luego desde la Comisión de Eficiencia Energética y Responsabilidad Ambiental del CAPBA10.

Hacia fines del año 2015, se dio reactivación a la Comisión de Desarrollo Sustentable (CDS) del CAPBA1. En ello, se propuso enlazar contenidos y actividades en relación a la arquitectura ambiental y energéticamente consciente, incluidos en el gran paradigma de la sustentabilidad. Desde un abordaje general, se propuso trabajar en la relación complementaria de la CDS con el resto de las Comisiones del CAPBA1; y desde lo específico se focalizó en el Desarrollo Sustentable centrado en la Eficiencia Energética Edilicia, fundamentados en el Convenio marco de cooperación (SSP y CAPBA) y la vigencia de la Ley ํ13059 de Eficiencia Energética.

La reactivación de la CDS, coordinada por el Dr. Arq. Lucas Rodríguez, comprendió la necesidad de abordar tres instancias principales e interdependientes: capacitación y difusión, asesoramiento técnico y relaciones institucionales. En tónica con la instancia de capacitación y difusión, se destacan los cursos de capacitación profesional titulados "El recurso energético en los edificios: orientación, ventilación y aislación" en los cuales se abordaron los temas introductorios y criterios básicos de la arquitectura ambiental y energéticamente consciente desde una mirada local. En relación al asesoramiento técnico, se propuesto la construcción de un espacio de asesoramiento en la temática de la Eficiencia Energética (EE) y las Energías Renovables (ER), desde el marco institucional del CAPBA1. En tanto a la instancia de relaciones institucionales, esta plantea el abordaje de los vínculos interinstitucionales para el establecimiento y regulación de las condiciones tecnológico-constructivas, termo-energéticas y de habitabilidad mínimas; en lo cual se presentó una propuesta según tres etapas: observación durante el visado, observación durante el permiso de obra, cumplimiento de la Ley № 13059 como requisito obligatorio.

A partir del 2016, el Instituto de Estudios Urbanos del CAPBA1 reestructuró su organización, afianzando las actividades de cada Comisión desde un proyecto institucional integrador. Este hecho, junto al cambio de residencia de Lucas Rodríguez (quien se muda desde La Plata a la ciudad de Tres Arroyos) redefinió los proyectos y proyecciones de la CDS hacia otras dimensiones de sustantivo interés dentro del desarrollo sustentable; pasando a la coordinación del Esp. Arq. Roberto Berardi.

Al respecto, estas redefiniciones habilitaron la posibilidad de expandir las zonas de trabajo en el propio marco del CAPBA: desde el CAPBA1, se ampliaron las líneas temáticas hacia el foco en el etiquetado energético edilicio. $Y$ desde el CAPBA10, se dio continuidad a los proyectos iniciales del autor, participando desde Tres Arroyos, Bahía Blanca y ciudades de la región. Al respecto, a inicios del año 2017, se propuso la creación de la Comisión de eficiencia energética y responsabilidad ambiental (CEERA) desde el CAPBA10, coordinado por los arquitectos Mario C. Rasquete, José Luis Fernández y Lucas Rodríguez, para abordar temas de la arquitectura sustentable desde la energía y el medio ambiente en el hábitat urbano.

Por lo tanto, en continuidad de los proyectos iniciados en 2015, la CEERA del CAPBA10 propuso abordar acciones en relación a la mejora de la calidad de habitabilidad en las construcciones arquitectónicas, trabajando en la eficiencia energética edilicia como estrategia prioritaria; a través de aportes de capacitación y asesoramientos técnicos (general y específico), acuerdos 
interinstitucionales en diversas temáticas afines y difusión de la producción de los partícipes del CAPBA en sus diversos distritos, exponiendo trabajos con particular preocupación por la eficiencia energética edilicia y una visión general de la sustentabilidad en su basto alcance. Al respecto, se desarrollaron numerosas charlas y cursos de capacitación técnica que alcanzaron los municipios de Bahía Blanca, Tres Arroyos, Pigüé, Puan, Darragueira, Suarez, entre otros.

La intención de estas jornadas, de carácter abierto y gratuito, se centró no sólo en la capacitación técnica respecto de los requerimientos de la Ley 13059 sino también en la oportunidad de generar un espacio de diálogo y reflexión entre los actores del sector privado y público, para comenzar a construir líneas de acción conjuntas que permitan la viabilización efectiva de las buenas prácticas de eficiencia energética edilicia.

\section{Etapa de formación académica}

Esta instancia, en incipiente desarrollo, busca complementar las propuestas hasta aquí descritas desde la inclusión del ámbito académico como momento anticipado en la construcción del hábitat urbano. En este sentido, se trabaja en el marco de la carrera de Arquitectura, Departamento de Geografía y Turismo (DGyT), Universidad Nacional del Sur (UNS) tanto en la carrera de grado como en la investigación académica.

A partir de 2018, los arquitectos José Luis Fernández y Lucas Rodríguez participan en un proyecto de grupos de investigación (PGI) titulado "Diagnóstico y propuesta de mejora de la eficiencia energética en la edificación educativa pública" (acreditación UNS, 2018-2021). En esta misma línea, se prevé la continuidad de trabajo sumando el tratamiento de la eficiencia energética en la edilicia construida desde el uso de la edificación educativa pública; proponiendo para la continuidad del proyecto el trabajo sobre protocolos de buenas costumbres y proyecciones desde la administración, aportando en dirección a políticas globales y particulares de sustentabilidad.

Paralelamente, los autores presentaron un proyecto de investigación académica para atender la instancia previa del diseño y tecnología, abordando el ejercicio de la sustentabilidad en la construcción arquitectónica, desde su proceso proyectual (Rodriguez y Fernandez, 2021). Recuperando acciones profesionales y académicas, y fusionando las áreas de experticia tanto en energía y tecnología (Rodriguez, 2015; 2017) como en didáctica de la arquitectura (Rodriguez, 2020), se busca reforzar la importancia de la concientización y difusión como instancia base para la aplicación de acciones y políticas de eficiencia energética; y así contribuir en la consolidación de una visión sustentable para la edificación y para el saber proyectual arquitectónico transferible a la formación de grado de los futuros arquitectos- (Rodriguez y Fernandez, 2019).

\section{CONCLUSIONES}

El refugio es un derecho universal. Por ende, el acceso a la vivienda es una temática que involucra la participación del Estado; comprendiendo no sólo a los organismos del gobierno sino también a las entidades privadas y a la sociedad en su conjunto. En esta tónica, la investigación descrita plantea un análisis crítico-reflexivo ante la concepción de las condiciones de esa "vivienda", trabajando en sus requerimientos de confort higrotérmico desde la eficiencia energética; reconociendo a su vez que la integración que propone la condición urbana nos obliga a evaluar la vivienda desde su interdependencia con la ciudad. Por lo tanto, se comprende que una acción efectiva para la eficiencia energética edilicia requiere del compromiso de todos los actores involucrados, cuyos accionares varías según la participación individual en el contexto urbano (fig. 2). 
La eficiencia energética en la vivienda como responsabilidad y beneficio conjunto

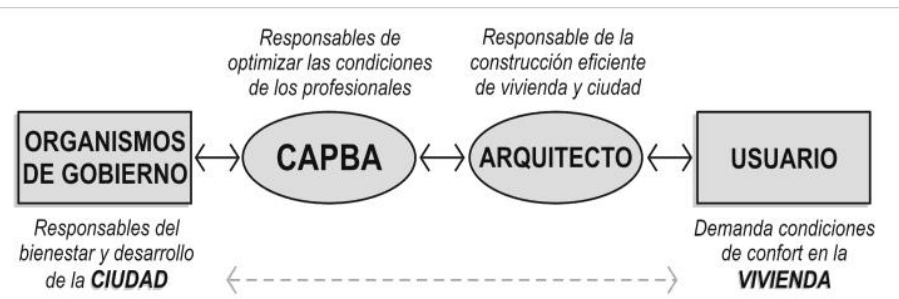

Figura 2: Actores intervinientes en el desarrollo de la vivienda y la ciudad. Fuente: Rodriguez, 2017

\section{-Participación en vivienda y ciudad desde los organismos de gobierno}

Tanto el gobierno nacional como los provinciales y municipales actúan en el par habitabilidadenergía desde la construcción y/o delimitación del parque residencial. En ello, las líneas principales de acción serían: la regulación de la construcción privada, la construcción de viviendas de interés social y el subsidio en los servicios energéticos. Por lo tanto, se comprende que los organismos de gobierno pueden aportar activamente, desde dos acciones posibles en promoción de la eficiencia energética edilicia en la provincia de Buenos Aires:

$1^{\circ}$ en obra a construir: incentivando las prácticas energéticas eficientes, estimulando la aplicación de normativas y leyes tales como la mencionada Ley №13059, en concordancia con el Decreto Nacional №140/07 y el №134/15.

$2^{\circ}$ en obra construida, financiar el reciclado (retrofitting) del parque residencial existente a las condiciones recomendadas por la Norma IRAM, y exigidas por la Ley 13059 para las viviendas nuevas, posiblemente mediante créditos blandos; reduciendo la demanda en el sector residencial, desafectando al sector industrial durante los períodos críticos y reduciendo las importaciones de combustibles fósiles. Todo lo cual se lograría con una inversión inicial que se recuperaría en períodos del orden de los 10 años (RODRIGUEZ et al., 2017).

Estas acciones aplicadas desde políticas nacionales (experiencias ya realizadas en gran parte de los países desarrollados luego de las crisis del petróleo) en consideración de una hipotética disminución en el consumo energético para calefacción del orden del $45 \%$ podrían reducir la demanda energética residencial en un $25 \%$, lo cual significaría una reducción en la matriz energética nacional del 7\% (RODRIGUEZ, 2015). Por citar un ejemplo (fig. 3), se menciona que la aplicación de mejora de la eficiencia energética en las viviendas de la ciudad de La Plata significaría el abastecimiento del total de las viviendas de Bahía Blanca. La aplicación sobre las viviendas de Bahía Blanca significaría el abastecimiento del doble de las viviendas de Tres Arroyos... La aplicación sobre las viviendas de la provincia de Buenos Aires (que representan el $39 \%$ del país) significa el total del interior de la provincia. Y la aplicación de esta reducción de consumo del $45 \%$ sobre la provincia de Buenos Aires es igual a la suma del consumo de las provincias de Córdoba, Santa Fe y Entre Ríos (INDEC, 2010; CHEVEZ et al., 2017).

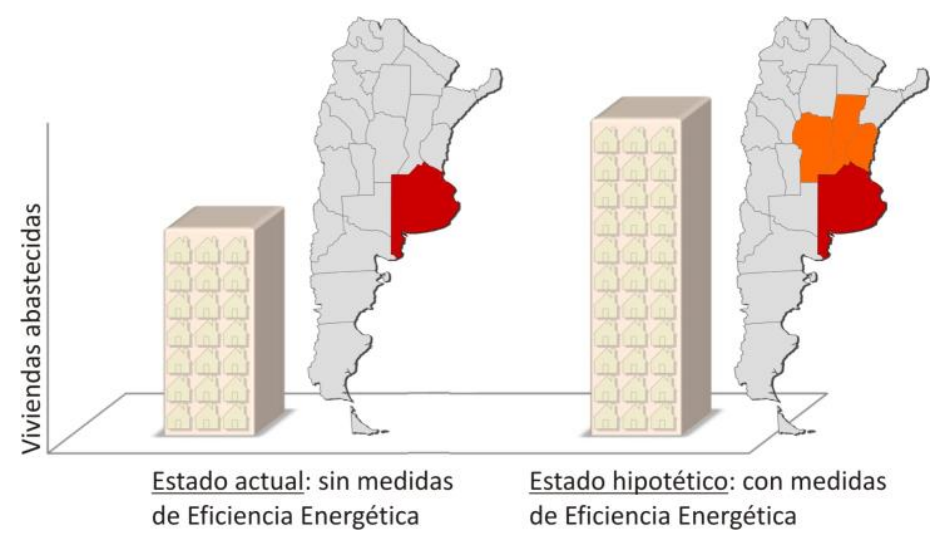

Figura 3: Abastecimiento de gas en viviendas, sin y con medidas de eficiencia energética. Fuente: Rodriguez, 2017 


\section{Participación en vivienda y ciudad desde el CAPBA}

El Colegio de Arquitectos de la Provincia de Buenos Aires, en su carácter de cuerpo colegiado, se comprende como órgano pertinente para la mejora de la vivienda y la ciudad, pudiendo colaborar desde la eficiencia energética edilicia a partir de su participación en la aplicación de la Ley №13039 en diversas formas:

- asesorando y defendiendo a los matriculados, en consideración del Artículo 6 de la Ley, que responsabiliza al profesional actuante por su incumplimiento.

- favoreciendo las correctas prácticas constructivas, con materiales acordes que favorezcan las condiciones dignas de habitabilidad

- colaborando con los municipios y la provincia en la aplicación de dicha Ley, vigente desde hace más de 7 años.

\section{Participación en vivienda y ciudad desde el profesional arquitecto}

Indiscutiblemente, nuestra participación profesional es de carácter prioritario en la construcción de la eficiencia energética edilicia en vivienda y ciudad, recordando que:

- es parte capital en nuestra tarea profesional el brindar un correcto asesoramiento en las diversas temáticas que incumben las construcciones arquitectónicas; en lo cual la calidad de habitabilidad higrotérmica a partir de una correcta calidad tecnológico-constructiva no sólo es fundamental, sino que, desde 2010, es exigida por Ley para el territorio de la Provincia de Buenos Aires.

- somos responsables de mantenernos actualizados en los temas que nos competen.

- somos responsables de la concientización al comitente de cada obra respecto de las condiciones de habitabilidad y de su relación con los consumos energéticos; alertando no sólo sobre los costos iniciales de construcción sino también los costos de mantenimiento posteriores. Desde esta mirada, es fundamental el actualizarnos en la temática, para dominar el contenido y de esta manera transmitir con confianza y seguridad lo que corresponde a una correcta calidad constructiva. Pues nadie discute que una viga de hormigón se construye con no menos de 3 hierros $\varnothing 8$ (es el reglamento y se respeta). Entonces ¿por qué es que sí discutimos si la vivienda debería llevar aislación?

\section{Participación en vivienda y ciudad desde el usuario}

Por último, remarcamos la necesaria participación del usuario para el establecimiento de una arquitectura energéticamente eficiente; quien es el principal afectado en la dependencia de energía auxiliar para lograr las condiciones de confort higrotérmico. Por lo tanto, se manifiestan beneficios en dos líneas principales:

- Habitabilidad: por las mejoras inmediatas en la calidad higrotérmica -estabilidad de temperatura, mejora en la calidad del aire y humedad- y la reducción o anulación de patologías en la construcción -como condensación, formación de hongos, mala calidad de aire interior-

- Energía y costo: por las reducciones en el consumo energético -tanto en invierno como en verano-, que son cada vez más inminentes en función de los actuales costos de las fuentes energéticas.

Al respecto, se entiende como necesario sustituir el preconcepto de "la aislación térmica como gasto extra" hacia "la aislación térmica como inversión"; pues, de hecho, al aislar "congelo" el costo de la energía que no se gastará, evitando la devaluación por inflación y los incrementos tarifarios. Dicho de otra manera, quien no aisló su vivienda tendrá que pagar esa energía que no ahorró más el sobrecosto del aumento sobre la energía auxiliar.

\section{Reflexiones finales}

A modo de corolario respecto de la investigación y las distintas acciones en eficiencia energética edilicia, resaltamos que la dirección propuesta no refiere al cumplimiento o no de un reglamento o una Ley, ni la exigencia de condiciones constructivas arbitrarias, sino hacia una comprensión 
global de la vivienda a favor de un correcto confort higrotérmico para el usuario y una mesurada demanda energética a nivel de ciudad.

Por lo tanto, para que las acciones en este sentido sean sólidas y coherentes, se requiere de una concientización que ayude a comprender que cada usuario tiene derecho a habitar en su vivienda con las condiciones dignas de confort; sabiendo a su vez que la integración de cada demanda individual genera un importante impacto a nivel urbano. Se requiere del compromiso de los profesionales encargados de construir la ciudad, asesorando tanto a los comitentes como a los órganos públicos pertinentes. Y se requiere de la participación del municipio y la provincia, para otorgar un marco mayor que asegure el beneficio para la sociedad en su conjunto. Hoy contamos con todos los puntos necesarios. Sólo resta el compromiso individual para aunar esfuerzos y producir líneas de acción concretas y sinérgicas entre el sector público y el privado, pues el beneficio está asegurado para todas las partes intervinientes (Rodriguez, 2017).

\section{REFERENCIAS BIBLIOGRÁFICAS}

Balance Energético Nacional (2020). Secretaría de Energía, Ministerio de Planificación Federal, Inversión pública $\quad y \quad$ servicios. Argentina. https://www.argentina.gob.ar/economia/energia/hidrocarburos/balances-energeticos. Visto el 30-082021.

Chévez, P.; Discoli, C.; Martini, I. (2017). "El consumo de gas natural por red. Análisis territorial y temporal". En revista Realidad económica, №308, 46. Pp. 117-137.

Discoli, C. (2009). Metodología para el diagnóstico urbano-energético-ambiental en aglomeraciones intermedias. El Caso del Gran La Plata. Libro elaborado sobre la Tesis doctoral del autor. Editorial Universitaria de La Plata, La Plata.

Guerrero, J. et al. (1983). "Conservación de energía. Estudio del consumo energético en viviendas de la zona templada húmeda". Informe final. IAS. Convenio № 166/82. La Plata, 1983.

Instituto Nacional de Estadística y Censos (INDEC) (2013); Intercambio Comercial Argentino, 23 de enero de 2013. Buenos Aires.

Instituto Nacional de Estadística y Censos (INDEC) (2010): Censo Nacional de Población, Hogares y Viviendas 2010. http://www.censo2010.indec.gov.ar/resultadosdefinitivos.asp, visto octubre 2014.

Rodríguez, L. (2020). "La evaluación formativa en Arquitectura. Aportes para la enseñanza desde la formación integral en los talleres FAU, UNLP”. Tesis Doctoral. DAU, FAU, UNLP. La Plata

Rodríguez, L. (2017). "Eficiencia energética edilicia: aportes de evaluación, aplicación y reflexión desde la vivienda a la ciudad". Primer premio categoría Difusión, en Premio Bianual a la Investigación Patrocinada, CAPBA 2017. Buenos Aires.

Rodríguez, L. (2015). "Instrumentación metodológica para el reciclado masivo de la edilicia residencial orientada a la eficiencia energética". Tesis de Doctorado en Ciencias- Área Energías Renovables, F.C.E., UNSa. Salta.

Rodríguez, L.; Díscoli, C.; Martini, I. (2017). Criterios y métodos para el reciclado edilicio con eficiencia energética. La Plata: Servicop.

Rodríguez, L.; Fernández, J. L. (2021). "Eficiencia energética y sustentabilidad como ejercicio de la coherencia: propuesta de investigación y docencia para el saber proyectual”. En Encuentro ARQUISUR 2021, Tucumán y Santiago de Chile.

Rodríguez, L.; Fernández, J. L. (2019). Material de cátedra, en "Teoría y Crítica hacia una Arquitectura Contemporánea y situada”, seminario de formación docente, DGyT, UNS. Bahía Blanca, 2019-2021.

Rosenfeld E., Czajkowski J. (1992). Catálogo de tipologías de viviendas urbanas en el área metropolitana de Buenos Aires. Su funcionamiento energético y bioclimático. Instituto de Estudios del Hábitat (IDEHAB), FAU, UNLP. La Plata

Samaja, J. (1994). Epistemología y Metodología. Elementos para una teoría de la investigación Científica. Editorial Universitaria de Buenos Aires. EUDEBA. Buenos Aires. Edición ampliada.

Viegas, G. (2010). "Evaluación del potencial energético e intervenciones de mejoramiento del entorno en áreas urbanas de media y baja consolidación. La ciudad de La Plata como caso de estudio". Tesis de Doctorado en Ciencias- Área Energías Renovables, F.C.E., UNSa. 